\title{
PPARG, GNG12, and CD19 are Potential Independent Predictors of Central Nerve Recurrence in Childhood Acute Lymphoblastic Leukemia
}

\author{
Shan Zhang \\ Nanchang University \\ Yansong Tu \\ University of Waterloo \\ Qianmiao Wu \\ Nanchang University \\ Huijun Chen \\ Nanchang University \\ Huaijun Tu \\ Second Affiliated Hospital of Nanchang University \\ Jian Li ( $\nabla$ thj127900@163.com) \\ Second Affiliated Hospital of Nanchang University
}

\section{Research Article}

Keywords: childhood acute lymphoblastic leukemia, central nervous system leukemia, TARGET, bioinformatics, biomarkers

Posted Date: November 1st, 2021

DOI: https://doi.org/10.21203/rs.3.rs-963371/v1

License: (c) (i) This work is licensed under a Creative Commons Attribution 4.0 International License. Read Full License 


\section{Abstract}

Objective: To identify biomarkers that can predict the recurrence of the central nervous system (CNS) in children with acute lymphoblastic leukemia (ALL), and establish a prediction model. Materials and

Methods: The transcriptome and clinical data collected by the Children's Oncology Group (COG) collaboration group in the Phase II study (use for test group) and Phase I study (use for validation group) of ALL in children were downloaded from the TARGET database. Transcriptome data were analyzed by bioinformatics method to identify core (hub) genes and establish a risk assessment model. Univariate Cox analysis was performed on each clinical data, and multivariate Cox regression analysis was performed on the obtained results and risk score. The children ALL phase I samples collected by the COG collaboration group in the TARGET database were used for verification.

Results: A total of 1230 differentially expressed genes were screened out between the CNS relapsed and non-relapsed groups. Univariate multivariate Cox analysis of 10 hub genes identified showed that PPARG (HR=0.78, 95\% Cl=0.67-0.91, $\mathrm{p}=0.007), \mathrm{CD} 19(\mathrm{HR}=1.15,95 \% \mathrm{Cl}=1.05-1.26, \mathrm{p}=0.003)$ and $\mathrm{GNG} 12$ $(\mathrm{HR}=1.25,95 \% \mathrm{Cl}=1.04-1.51, \mathrm{p}=0.017)$ had statistical differences. The risk score was statistically significant in univariate $(\mathrm{HR}=3.06,95 \% \mathrm{Cl}=1.30-7.19, \mathrm{p}=0.011)$ and multivariate $(\mathrm{HR}=1.81,95 \% \mathrm{Cl}=1.16-2.32, \mathrm{p}=0.046)$ Cox regression analysis. The survival analysis results of the high and low-risk groups were different when the validation group was substituted into the model $(\mathrm{p}=0.018)$. In addition, the CNS involvement grading status at first diagnosis CNS3 vs. CNS1 $(\mathrm{HR}=5.74,95 \% \mathrm{Cl}=2.01-16.4, \mathrm{p}=0.001)$, $\mathrm{T}$ cell vs $\mathrm{B}$ cell $(\mathrm{HR}=1.63,95 \% \mathrm{Cl}=1.06-2.49, \mathrm{p}=0.026)$ were also statistically significant.

Conclusions: PPARG, GNG12, and CD19 may be predictors of CNS relapse in childhood ALL.

\section{Introduction}

Childhood acute lymphoblastic leukemia (ALL) is one of the most common primary malignant tumors in children and adolescents, accounting for about $35 \%$ of all tumors in children and more than $75 \%$ of all childhood acute leukemia cases ${ }^{[1]}$. Although most ALL kids frequently achieve complete remission, about $3-8 \%$ of children with ALL suffer from relapse in the central nervous system (CNS). Those suffering from CNS leukemia (CNSL) are believed to have poorer outcomes than CNS-negative kids ${ }^{[2,3]}$. Traditional intrathecal injection of chemotherapeutic drugs and radiotherapy has been associated with side effects and CNS relapse after remission. In addition, the majority of CNS relapse occurs in patients who were CNS-negative at initial diagnosis ${ }^{[4]}$. Therefore, exploring new favorable biomarkers for CNS relapse may significantly improve the early diagnosis and treatment in childhood ALL.

Studies have reported that gender, hepatomegaly, central nervous system status and age ${ }^{[5]}$, neuronal-glial antigen-2 (NG2) expression ${ }^{[6]}$, and CNS microenvironment ${ }^{[7]}$ are all associated with CNS relapse of childhood ALL. Another study showed that the up-regulation of the $P B \times 1$ gene in B-cell leukemia in mouse CNS microenvironment could enhance the chemotherapy-resistance and self-renewal characteristics of leukemia cells ${ }^{[8]}$. However, as these studies were based only on clinical data or animal experiments, there is still no independent predictor of CNS relapse in childhood ALL.

The aim of this study was to identify biomarkers that can predict the relapse of CNS in childhood ALL and establish a prediction model to provide early preventive therapy and targeted therapy for the childhood ALL at high risk of CNS relapse.

\section{Materials And Methods}

\section{Clinical Data and preprocessing}

We downloaded clinical and transcriptome data for Phase II of the ALL program from the TARGET database (https://ocg.cancer.gov/programs/target). The data obtained from the database were divided into CNS relapsed group and non-relapsed group based on the presence or absence of CNS relapse. The inclusion and exclusion criteria for the two groups of data were: (1) patients were younger than 18 years old at the time of diagnosis; (2) complete transcriptome data were obtained in both groups; (3) clinical information should include complete relapse-free survival, CNS relapse status, etc.; (4) relapsed group included patients that had only CNS relapse and no other site relapse at the end of the follow-up ;the non-relapsed group refers to patients with no recurrence in any part by the end of follow-up

\section{Differentially expresses genes (DEGs) and enrichment analysis}

The edge $\mathrm{R}$ was used to identify DEGs between the two groups (relapsed group vs. non-relapsed group). A false discovery rate (FDR) adjusted $\mathrm{p}<0.05$ combined with a simultaneous absolute value of $\geq 2$ for logFC was set as the threshold for DEGs identification. The results of the differential analysis were visualized using gplots on the volcanic map and heat map. The differential genes were imported into the Biomart application of the Ensembl website for gene ID conversion. GO function enrichment analysis (annotated from the biological process (BP), cell composition (CC), molecular function (MF)), and KEGG pathway analysis was performed using the David website. The STRING was used for the analysis of protein interactions encoded by differential genes; the credibility $>0.7$ was used as a cut-off value to plot the Protein-protein interaction network (PPI).

Twelve topological analysis methods, including Degree Edge Percolated Component (EPC), Maximum neighbourhood component (MNC), Density of Maximum Neighborhood Component (DMNC), and Maximal Clique Centrality (MCC) etc, were provided by the CytoHubba plug-in in the Cytoscape software. These methods were used to analyze protein interaction networks. The top 10 scoring genes obtained by various algorithms were counted for frequency, and the top 10 genes with the highest occurrence frequency were regarded as hub genes.

\section{Survival analysis and stepwise backward regression, ROC curve}


The top 10 DEGs with the highest occurrence frequency were used as hub genes for univariate Cox regression analysis using Survival R packet. Multivariate Cox regression included statistically significant DEGs, and the stepwise backward regression was used to establish the optimal model. Finally, the risk value was calculated using the following formula: the risk score $=\left(\beta 1^{*}\right.$ expression level of $\left.P P A R G\right)+(\beta 2 *$ expression level of $C D 19)+\left(\beta 3^{*}\right.$ expression level of GNG12). The ROC curves of 3-5 years were drawn every six months in batches using the survival ROC package. ROC curve at the time point with the maximum AUC was selected to intercept the turning point, which was used as the cut-off value of the risk score. The research subjects were divided into high and low-risk groups based on the cut-off value of the risk score. The Kaplan-Meier survival curves for the cases predicted to have low or high risk were produced to verify whether the two groups showed statistical significance.

\section{Validation}

The data collected by the COG collaboration group in the TARGET database for phase I of the study on childhood ALL were used to verify. The expression levels of the three target genes identified in the study were included in the model, and the risk score was calculated. The high-risk group and the low-risk group were also divided, and survival analysis was conducted to verify the accuracy of the model. Finally, to further validate whether the biomarkers' prediction was independent of other clinical variables, univariate and multivariate Cox regression, stratified analyses were conducted.

\section{Statistical and analytical software}

R3.6.0 was used for all data analysis and mapping. Gene ID conversion through Ensembl (https://grch37.ensembl.org/info/data/biomart/index.html). Enrichment analysis was conducted by the David website (https://david.ncifcrf.gov/tools.jsp). PPI plot was drawn by String website String (https://string$\mathrm{db}$.org/). Hub gene was determined by Cytoscape 3.8.2. P-value of $<0.05$ was considered as statistical significance, and all reported $p$-values were two-sided.

\section{Results}

\section{Patient characteristics}

Clinical information of the subjects in the relapsed group and non-relapsed group were described by means and percentages. A total of 278 subjects were included in this study, with an average age of $8.7 \pm 4.6$ years. The time of diagnosis was from 2001 to 2011 , and the follow-up period was from 2005 to 2016(the mean follow-up time was 6.3 years); the male to female ratio was 3:7. Based on the presence or absence of CNS relapse, the data were divided into CNS relapsed and non-relapsed groups, with 28 patients in the CNS relapsed group and 250 patients in the non-relapsed group. Due to many missing values of the ETV6-RUNX1 fusion gene and only 4 positive cases, and since only one case of TCF3-PBX1 and BCR-ABL 1 genes were positive, subsequent studies were not included in the analysis (Table 1).

\section{DEGs analysis}

A total of 1230 differential genes were screened, including 685 up-regulated and 545 down-regulated genes. G plots were used to visualize the different analysis results, as shown in Figure 1 and Figure 2.

\section{Identification of Differentially Expressed Genes and Functional Enrichment Analysis}

The results showed that these differential genes were mainly involved in biological processes such as antigen processing and positive regulation of $\mathrm{T}$ cell aggregation, angiogenesis, and cell adhesion, migration, and proliferation of presenting cells. The cell components were mainly concentrated on cell surface, transport vesicle membrane, endocytic vesicle membrane, and involved in the composition of the MHC class II protein complex. As for molecular function, the results showed that genes were mainly involved in $\mathrm{MHC}$ class II receptor activity, growth factor activity, signal transduction activity, calmodulin-binding activity, etc. $(p<0.05)$ (Figure $3 A)$. KEGG pathway analysis showed that these genes were mainly involved in antigen processing and presentation-related pathways, PI3K-Akt signaling pathway, Ras signaling pathway, and cancer-related signaling pathways $(p<0.05)$ (Figure 3B).

\section{Protein interaction network (PPI) and hub gene identification}

A total of 580 proteins and 647 edges were obtained with a cut-off value of credibility $>0.7$. The average interaction between each node was 2.23 , and the aggregation coefficient was 0.315 , as shown in Figure 4.

Frequency statistics were carried out for the top 10 genes scored by various algorithms, and the top 10 genes (NPY, IL1B, IL6, BMP2, PPARG, CD19, GNG13, CD40, GNG7, GNG12) with the highest occurrence frequency were regarded as hub genes (Table 2 and Figure 5).

\section{PPARG, CD19, and GNG12 were obtained by Cox regression analysis}

Univariate Cox regression analysis was performed for the top 10 hub genes using Survival packet (recurrence as the outcome variable, time without recurrence as time-variable). The results showed that all the 10 hub genes were statistically significant, and they were included in multivariate Cox regression analysis (Table 3).

The results of multivariate analysis showed that there were three genes with significant differences, namely PPARG, CD19, and GNG12 (Table 3). In the CNS relapsed group, PPARG showed the lowest expression, while CD19 and GNG12 had the highest expression. The risk model was obtained according to the regression results as follows:

risk score $=P P A R G^{\star}(-0.19009)+C D 19^{\star}(0.13794) *+G N G 12^{\star}(0.22447)$ 
For the obtained risk model, the ROC curves at multiple time points across 3-5 years were done in batches to compare and screen out the time point at which the maximum AUC was obtained. The results showed that the model established in this study has a good predictive ability in predicting the 5-year relapse rate (AUC max=0.75) (Figure 6).

We calculated the risk score of the 278 research objects. The true positive and false positive difference maximum points were taken as cut-off of the risk score. Values above this score were defined as high-risk group, and below this score as a low-risk group. Kaplan-Meier survival curve and log-rank test were adopted to appraise the association of the overall survival (Figure 7).

In order to verify the accuracy of the model, we used transcriptome and clinical data collected from the COG collaboration group in the Target database for the phase of childhood ALL for verification. The expression levels of the three target genes identified in the study were included in the model, and the risk values were calculated. The risk groups were also divided into high and low-risk groups, and survival analysis (K-M curve) was performed. Our results proved that the established model has a good predictive ability $(p=0.018)$ (Figure 8).

\section{The risk score can be used as an independent prognostic factor}

To judge whether the model's risk score can be used as an independent predictor of a childhood ALL relapse of CNS, we conducted Cox regression analyses with clinical data (Table 4). The results showed that the risk score was statistically significant in univariate $(\mathrm{HR}=3.06,95 \% \mathrm{Cl}=1.30-7.19, \mathrm{p}=0.011)$ and multivariate $(\mathrm{HR}=1.81,95 \% \mathrm{Cl}=1.16-2.32, \mathrm{p}=0.046)$ Cox regression analysis. CNS3 vs. CNS1 $(\mathrm{HR}=5.74,95 \% \mathrm{Cl}=2.01-16.4, \mathrm{p}=0.001)$ and $\mathrm{T}$ cell vs. $\mathrm{B}$ cell $(\mathrm{HR}=1.63,95 \% \mathrm{Cl}=1.06-2.49, \mathrm{p}=0.026)$ were significantly different between the two groups; while no difference was found for patients' gender (HR=1.20, $95 \% \mathrm{Cl}=0.72-2.02, \mathrm{p}=0.490)$.

We stratified the expression of the CD19 gene according to the source of T cells and B cells. The results showed that this gene differed only in B lymphocytic leukemia( $(p=0.018)$, as shown in Table 5.

\section{Discussion}

The CNS is a common site for relapse of ALL. Yet, considering that most drugs cannot pass through the brain blood-brain barrier ((BBB), the treatment of CNS leukemia is still very challenging ${ }^{[9]}$. Therefore, identifying new potential biomarkers that could predict CNS relapse in children is extremely important. In this study, bioinformatics correlation analysis was used to identify biomarkers, revealing that PPARG, GNG12, and CD19 were independent prognostic factors for childhood ALL with CNS relapse.

PPARG gene is a member of the nuclear receptor family, which encodes peroxisome proliferative activated receptor gamma (PPARY), PPARy was first identified in adipocytes, as a transcription factor, the PPARy receptor binds to its ligand and inhibits tumor proliferation, metastasis, angiogenesis, and promotes apoptosis and immune regulation by activating signaling pathways of different tumor cells and tumor stem cells ${ }^{[10]}$. For example, PPARy can induce apoptosis by inhibiting the PI3K/Akt signaling pathway, PPARY ligand can induce the apoptosis of leukemia cells, and can be potentially used to treat hematologic malignancies, especially leukemia ${ }^{[11]}$. In our study, we found a low expression of PPARG in childhood ALL with CNS relapse. Moreover, KEGG analysis showed activation of the PI3K/Akt signaling pathway, which suggests that PPARG might regulate CNS relapse in ALL children through the PI3K/Akt signaling pathway. Boyd et al ${ }^{[12]}$ found that the injection of PPARs antibody agonist could induce adipogenesis in bone marrow tissue in mice, thereby saving healthy hematopoietic cell formation during the inhibition of leukemia cell growth. The most common way leukemia cells invade the central nervous system is from the bone marrow of the skull to the subarachnoid space via dural bridging vein ${ }^{[13]}$. When the expression of the PPARG gene is low, this induction function of PPARs is inhibited, which might in turn lead to the CNS relapse of ALL in children.

The GNG12, a gene encoding Gy12, a member of the G12 subfamily of heterotrimeric $\mathrm{G}$ proteins, is involved in the regulation of expression and distribution of tightly connected endothelial cells ${ }^{[14]}$. The BBB is mainly composed of tight junctions (TJ) found between endothelial cells, which limit the passage of substances in the brain ${ }^{[15]}$. Donato et al found that heterotrimer $\mathrm{G}$ protein has an important role in TJ's formation and regulation, which is regulated through the intercellular signaling pathway and has an important influence on the BBB structure ${ }^{[16]}$. It has also been found that Gy12 preferentially interacts with adrenergic receptors in activated central synapses ${ }^{[17]}$. Adrenergic receptors are involved in the regulation of protein kinase $A(P K A)$ and $c A M P{ }^{[18]}$. The BBB appears to be highly sensitive to PKA levels and opens when cAMP and PKA levels decrease ${ }^{[19]}$. This may result in leukemic cells crossing the blood-brain barrier more easily and invading the CNS.

CD19 play a key role in maintaining the balance of humoral immunity, antigen induction, and immune tolerance. The relationship between $C D 19$ and ALL has not been fully defined. Still, it is well known that CD19 is the most densely expressed antigen on B cells' surface in ALL patients and is a highly specific target for drug development ${ }^{[20]}$. In particular, CAR T cells can penetrate the BBB into the CNS, killing leukemic cells in the cerebrospinal fluid ${ }^{[21]}$. Jin MY et al $^{[22]}$ reported two cases of acute leukemia patients with CNSL, which showed positive CD19 expression in tumor cell immunotyping and remission after antiCD19 CAR T cell therapy. In addition, studies have reported the efficacy of anti-CD19 CAR T cell therapy in patients with ALL-CNSL ${ }^{[23-26]}$. These treatments further revealed the relationship between $C D 19$ and $C N S L$, and also verified the high relapse risk of CNS in patients with high expression of the $C D 19$ gene in this study. Meanwhile, stratified analysis results show that this difference only appeared in B-ALL, which also verified the reliability of the results of this study. In other words, the high expression of the CD19 gene is not only a marker of B-ALL but may also lead to CNS relapse when its expression level reaches a certain level.

In this study, the cell composition, molecular function, biological process, and KEGG pathway corresponding to the DEGs were enriched and analyzed. These results revealed a related pathway leading to the relapse of CNS, in which calmodulin-binding was related to the molecular composition and signal regulation 
of the tight junction of the BBB. Some researchers have reported that leukemia cells enter CNS via vascular transport, which involves the interaction between A6 integrin and laminin and can be blocked by specific PI3K inhibitors ${ }^{[27]}$. Our results further suggest that CNS relapse in childhood ALL is related to the PI3KAkt signaling pathway. In addition, cell adhesion molecules (CAMs), antigen processing and presentation, and neuroactive ligand-receptor interactions provide upstream and downstream factors and targets for future studies on CNSL recurrence, which should be further explored.

The results of our study confirmed that CNS grade 3 at initial diagnosis was a risk factor for CNS relapse, which was consistent with previous studies ${ }^{[6]}$. Our data also suggested that T lymphocytic leukemia in children was more likely to lead to CNS relapse than B lymphocytic leukemia, which was also consistent with previous relevant reports ${ }^{[28-30]}$. This study failed to prove that hyperleukocytosis and male in patients were high-risk factors for CNS relapse, which were inconsistent with previous studies ${ }^{[5,31]}$, We speculate that it may be due to sample differences and should be further clarified by future studies.

To sum up, this study verified that CNS3 classification and the types of T lymphocytes are risk factors for CNS relapse of childhood ALL. We found that PPARG, GNG12 and CD19 can be used as independent predictors of CNS relapse in childhood ALL, yet, more studies are needed to confirm these findings. Our results may provide a theoretical basis for further studies to elucidate the molecular mechanism of CNS relapse in childhood ALL and provide more therapeutic targets for future clinical interventions.

\section{Declarations}

Author's ContributionロShan Zhang, Yansong Tu, Qianmiao Wu, Huijun Chen, Huaijun Tu and Jian Li. wrote the main manuscript text and prepared figures 1-8 and tables 1-5. All authors reviewed the manuscript.

Ethics and dissemination: This study does not need to be reviewed by the Ethics Committee, because this paper is not a clinical study or a related experimental study, and this paper is only a literature study.

Availability of Data and Materials : Our study received written approval from the $\mathrm{NCl}$ Office of Cancer Genomics. The results published here are in whole based upon data generated by the Therapeutically Applicable Research to Generate Effective Treatments (https://ocg.cancer.gov/programs/target) initiative, phs000218. The data used for this analysis are available at https://portal.gdc.cancer.gov/projects. We acknowledge the TARGET database for providing their platforms and contributors for uploading their meaningful datasets.

Funding: This work was supported by the [Natural Science Foundation of Jiangxi Province \#1] under Grant [number 20171BAB205045] and [the National Natural Science Foundation of China \#2] under Grant [number 8176010176].

Conflict of interest]We declare that we do not have any commercial or associative interest that represents a conflict of interest in connection with the work submitted.

\section{References}

[1] Mueller KT, Maude SL, Porter DL, et al. Cellular kinetics of CTL019 in relapsed/refractory B-cell acute lymphoblastic leukemia and chronic lymphocytic leukemia [J]. Blood, 2017, 130(21): 2317-25.

[2] Ikonomidou C. Cerebrospinal Fluid Biomarkers in Childhood Leukemias [J]. Cancers (Basel), 2021, 13(3):

[3] Saeedi A, Baghestani A, Khadem Maboudi A, et al. Determining the Significant Prognostic Factors for the Recurrence of Pediatric Acute Lymphoblastic Leukemia Using a Competing Risks Approach [J]. Iran J Med Sci, 2020, 45(4): 304-10.

[4] Kinjyo I, Bragin D, Grattan R, et al. Leukemia-derived exosomes and cytokines pave the way for entry into the brain [J]. J Leukoc Biol, 2019, 105(4): 741-53.

[5] Matloub Y, Lindemulder S, Gaynon PS, et al. Intrathecal triple therapy decreases central nervous system relapse but fails to improve event-free survival when compared with intrathecal methotrexate: results of the Children's Cancer Group (CCG) 1952 study for standard-risk acute lymphoblastic leukemia, reported by the Children's Oncology Group [J]. Blood, 2006, 108(4): 1165-73.

[6] Prieto C, López-Millán B, Roca-Ho H, et al. NG2 antigen is involved in leukemia invasiveness and central nervous system infiltration in MLL-rearranged infant B-ALL [J]. Leukemia, 2018, 32(3): 633-44.

[7] Gossai NP, Gordon PM. The Role of the Central Nervous System Microenvironment in Pediatric Acute Lymphoblastic Leukemia [J]. Front Pediatr, $2017,5(90$.

[8] Gaynes JS, Jonart LM, Zamora EA, et al. The central nervous system microenvironment influences the leukemia transcriptome and enhances leukemia chemo-resistance [J]. Haematologica, 2017, 102(4): e136-e9.

[9] Lenk L, Alsadeq A, Schewe DM. Involvement of the central nervous system in acute lymphoblastic leukemia: opinions on molecular mechanisms and clinical implications based on recent data [J]. Cancer Metastasis Rev, 2020, 39(1): 173-87.

[10] Janani C, Ranjitha Kumari BD. PPAR gamma gene-a review [J]. Diabetes Metab Syndr, 2015, 9(1): 46-50.

[11] Yousefnia S, Momenzadeh S, Seyed Forootan F, et al. The influence of peroxisome proliferator-activated receptor Y (PPARY) ligands on cancer cell tumorigenicity [J]. Gene, 2018, 649(14-22. 
[12] Boyd A, Bhatia M. Acute myeloid leukaemia disrupts endogenous myelo-erythropoiesis by compromising the adipocyte bone marrow niche [J]. Nature Cell Biology, 2017, 19(11): 1336-47.

[13] Zhou F, Wen Y, Jin R, et al. New attempts for central nervous infiltration of pediatric acute lymphoblastic leukemia [J]. Cancer Metastasis Rev, 2019, 38(4): 657-71.

[14] Li J, Jin C, Zou C, et al. GNG12 regulates PD-L1 expression by activating NF-kB signaling in pancreatic ductal adenocarcinoma [J]. FEBS Open Bio, 2020, 10(2): 278-87.

[15] Langen UH, Ayloo S, Gu C. Development and Cell Biology of the Blood-Brain Barrier [J]. Annu Rev Cell Dev Biol, 2019, 35(591-613.

[16] González-Mariscal L, Raya-Sandino A, González-González L, et al. Relationship between G proteins coupled receptors and tight junctions [J]. Tissue Barriers, 2018, 6(1): e1414015.

[17] Yim YY, Betke KM, McDonald WH, et al. The in vivo specificity of synaptic G $\beta$ and Gy subunits to the a(2a) adrenergic receptor at CNS synapses [J]. Sci Rep, 2019, 9(1): 1718.

[18] Shi Q, Li M, Mika D, et al. Heterologous desensitization of cardiac $\beta$-adrenergic signal via hormone-induced $\beta A R /$ arrestin/PDE4 complexes [J]. Cardiovasc Res, 2017, 113(6): 656-70.

[19] Fukuda S, Nakagawa S, Tatsumi R, et al. Glucagon-Like Peptide-1 Strengthens the Barrier Integrity in Primary Cultures of Rat Brain Endothelial Cells Under Basal and Hyperglycemia Conditions [J]. J Mol Neurosci, 2016, 59(2): 211-9.

[20] Pacenta HL, Laetsch TW, John S. CD19 CAR T Cells for the Treatment of Pediatric Pre-B Cell Acute Lymphoblastic Leukemia [J]. Paediatr Drugs, 2020, 22(1): 1-11.

[21] Maus MV, Grupp SA, Porter DL, et al. Antibody-modified T cells: CARs take the front seat for hematologic malignancies [J]. Blood, 2014, 123(17): 2625-35.

[22] Jin MY, Han Y, Liu YJ, et al. [Treatment of central nervous system leukemia with CD19-chimeric antigen receptor T-cell immunotherapy: two cases report and literature review] [J]. Zhonghua Xue Ye Xue Za Zhi, 2018, 39(8): 650-3.

[23] Nair S, Wang JB, Tsao ST, et al. Functional Improvement of Chimeric Antigen Receptor Through Intrinsic Interleukin-15Ra Signaling [J]. Curr Gene Ther, 2019, 19(1): 40-53.

[24] Maude SL, Frey N, Shaw PA, et al. Chimeric antigen receptor T cells for sustained remissions in leukemia [J]. N Engl J Med, 2014, 371(16): 1507-17.

[25] Lee DW, Kochenderfer JN, Stetler-Stevenson M, et al. T cells expressing CD19 chimeric antigen receptors for acute lymphoblastic leukaemia in children and young adults: a phase 1 dose-escalation trial [J]. Lancet, 2015, 385(9967): 517-28.

[26] Hu Y, Sun J, Wu Z, et al. Predominant cerebral cytokine release syndrome in CD19-directed chimeric antigen receptor-modified T cell therapy [J]. J Hematol Oncol, 2016, 9(1): 70.

[27] Yao H, Price TT, Cantelli G, et al. Leukaemia hijacks a neural mechanism to invade the central nervous system [J]. Nature, 2018, 560(7716): 55-60.

[28] Goldberg JM, Silverman LB, Levy DE, et al. Childhood T-cell acute lymphoblastic leukemia: the Dana-Farber Cancer Institute acute lymphoblastic leukemia consortium experience [J]. J Clin Oncol, 2003, 21(19): 3616-22.

[29] Schrappe M, Reiter A, Ludwig WD, et al. Improved outcome in childhood acute lymphoblastic leukemia despite reduced use of anthracyclines and cranial radiotherapy: results of trial ALL-BFM 90. German-Austrian-Swiss ALL-BFM Study Group [J]. Blood, 2000, 95(11): $3310-22$.

[30] Crist W, Shuster J, Look T, et al. Current results of studies of immunophenotype-, age- and leukocyte-based therapy for children with acute lymphoblastic leukemia. The Pediatric Oncology Group [J]. Leukemia, 1992, 6 Suppl 2(162-6.

[31] Cancela CS, Murao M, Viana MB, et al. Incidence and risk factors for central nervous system relapse in children and adolescents with acute lymphoblastic leukemia [J]. Rev Bras Hematol Hemoter, 2012, 34(6): 436-41.

\section{Tables}

\section{Table 1. Patients' characteristics}




\begin{tabular}{|c|c|c|c|}
\hline Term & $\begin{array}{l}\text { total } \\
(n=278)\end{array}$ & $\begin{array}{l}\text { relapsed } \\
(n=28)\end{array}$ & non-relapsed $(n=250)$ \\
\hline Age (years $₫$ mean $\pm S D)$ & $8.7 \pm 4.6$ & $7.4 \pm 5.0$ & $8.8 \pm 4.5$ \\
\hline \multicolumn{4}{|l|}{ Gender (n, \%) } \\
\hline Male & $84(30.2)$ & $13(46.4)$ & $71(28.4)$ \\
\hline Female & $194(69.8)$ & $15(53.6)$ & $179(71.6)$ \\
\hline \multicolumn{4}{|l|}{ Race $(n, \%)$} \\
\hline White & $205(73.7)$ & $24(85.7)$ & $181(72.4)$ \\
\hline Others ${ }^{a}$ & $73(26.3)$ & $4(14.3)$ & $69(27.6)$ \\
\hline \multicolumn{4}{|l|}{$\operatorname{Race}^{b}(n, \%)$} \\
\hline Hispanic or Latino & 49 (18.9) & $8(28.6)$ & $41(17.7)$ \\
\hline Not & $210(81.1)$ & $20(71.4)$ & $190(82.3)$ \\
\hline Relapse-free survival (months) & $76.9 \pm 26.4$ & $60.6 \pm 32.1$ & $78.7 \pm 25.1$ \\
\hline \multicolumn{4}{|l|}{ Survival status $(\mathrm{n}, \%)$} \\
\hline Alive & $261(93.9)$ & $11(39.3)$ & $250(100)$ \\
\hline Dead & $17(6.1)$ & $17(60.7)$ & $0(0)$ \\
\hline \multicolumn{4}{|l|}{ WBC count $\left(10^{9} / \mathrm{L}\right)(\mathrm{n}, \%)$} \\
\hline$>100$ & $128(46.0)$ & $12(42.9)$ & $128(46.0)$ \\
\hline$(10,100]$ & $124(44.6)$ & $13(46.4)$ & $124(44.6)$ \\
\hline$\leq 10$ & $26(9.4)$ & $3(10.7)$ & $26(9.4)$ \\
\hline Day-29 MRD & $0.51 \pm 1.3$ & $0.51 \pm 1.3$ & $0.45 \pm 1.3$ \\
\hline \multicolumn{4}{|l|}{ Day-29 MRD ${ }^{b}(n, \%)$} \\
\hline$>0$ & $98(35.5)$ & $12(42.9)$ & $86(34.7)$ \\
\hline 0 & $178(64.5)$ & $16(57.1)$ & $162(65.3)$ \\
\hline \multicolumn{4}{|l|}{ CNS classification ${ }^{b}(\mathrm{n}, \%)$} \\
\hline CNS1 & $197(71.1)$ & $18(64.2)$ & 179 (71.9) \\
\hline CNS2 & $61(22.0)$ & $5(17.9)$ & $56(22.5)$ \\
\hline CNS3 & $19(6.9)$ & 5 (17.9) & $14(5.6)$ \\
\hline \multicolumn{4}{|l|}{ ETV6 RUNX1 ${ }^{\mathrm{b}}(\mathrm{n}, \%)$} \\
\hline Negative & $122(96.8)$ & $16(84.2)$ & $106(99.1)$ \\
\hline Positive & $4(3.2)$ & $3(15.8)$ & $1(0.9)$ \\
\hline \multicolumn{4}{|l|}{ TCF3-PBX1 $1^{\mathrm{b}}(\mathrm{n}, \%)$} \\
\hline Negative & 199 (99.5) & $18(94.7)$ & $181(100)$ \\
\hline Positive & $1(0.5)$ & $1(5.3)$ & $0(0)$ \\
\hline \multicolumn{4}{|l|}{ BCR-ABL1(n, \%) } \\
\hline Negative & $277(99.6)$ & $28(100)$ & $249(99.6)$ \\
\hline Positive & $1(0.4)$ & $0(0)$ & $1(0.4)$ \\
\hline \multicolumn{4}{|l|}{ TRISOMY 410 Status $^{\text {b }}$} \\
\hline Negative & $221(96.5)$ & $23(100)$ & $198(96.1)$ \\
\hline Positive & $8(3.5)$ & 0 & $8(3.9)$ \\
\hline \multicolumn{4}{|l|}{ MLL Status $^{\mathrm{b}}(\mathrm{n}, \%)$} \\
\hline Negative & $227(95.4)$ & $26(100)$ & $201(94.8)$ \\
\hline
\end{tabular}




\begin{tabular}{|llll|}
\hline Positive & $11(4.6)$ & 0 & $11(5.2)$ \\
\hline BMA Blasts Day 8 & $20.3 \pm 26.3$ & $30.0 \pm 31.4$ & $19.2 \pm 25.6$ \\
\hline BMA Blasts Day 15 & $4.6 \pm 8.8$ & $7.4 \pm 16.8$ & $4.3 \pm 7.1$ \\
\hline BMA Blasts Day 29 & $0.7 \pm 1.2$ & $1.1 \pm 1.5$ & $0.7 \pm 1.1$ \\
\hline DNA Index $(\mathrm{n}, \%)$ & & & \\
\hline 1 & $228(83.8)$ & $20(76.9)$ & $208(84.6)$ \\
\hline$>1$ & $44(16.2)$ & $6(23.1)$ & $38(15.4)$ \\
\hline Cell of origin ${ }^{\mathrm{b}}(\mathrm{n}, \%)$ & & & \\
\hline B Cell & $74(27.1)$ & $13(50.0)$ & $61(24.7)$ \\
\hline T Cell & $199(72.9)$ & $13(50.0)$ & $186(75.3)$ \\
\hline
\end{tabular}

Table 2. Twelve Topological analysis methods of top 10 genes ranked by Degree

\begin{tabular}{|c|c|c|c|c|c|c|c|c|c|c|c|}
\hline MCC & DMNC & MNC & Degree & EPC & BottleNeck & EcCentricity & Closeness & Radiality & Betweenness & Stress & ClusteringCoeffici \\
\hline GNG13 & $\begin{array}{l}H L A- \\
D M A\end{array}$ & IL6 & IL6 & IL6 & IL6 & IL6 & IL6 & IL6 & IL6 & IL6 & SYT13 \\
\hline GNG7 & $H L A-D O A$ & GNG13 & GNG13 & CD19 & $I L 1 B$ & $I L 1 B$ & $I L 1 B$ & $I L 1 B$ & $N P Y$ & $N P Y$ & LOXHD1 \\
\hline GNG12 & $F 2 R L 3$ & GNG7 & GNG7 & $I L 1 B$ & $N R P 1$ & $N P Y$ & $B M P 2$ & $N P Y$ & $N R P 1$ & GNG13 & $H L A-D M A$ \\
\hline GNG11 & CYSLTR2 & CD19 & CD19 & $C D 40$ & PPARG & CCL $4 L 1$ & $N P Y$ & $B M P 2$ & CD19 & GNG7 & LDOC1 \\
\hline$B D K R B 2$ & $E D N R B$ & GNG12 & $C D 40$ & IRF4 & $N P Y$ & PPARG & CD19 & PPARG & $I L 1 B$ & PRKCG & $H L A-D O A$ \\
\hline$B D K R B 1$ & UTS2R & $C D 40$ & GNG12 & GNG13 & GNG13 & HPGDS & $E N G$ & ENG & PRKCG & $I L 1 B$ & CLIC5 \\
\hline$N P Y$ & $\begin{array}{l}H L A- \\
D R B 5\end{array}$ & GNG11 & IRF4 & $N P Y$ & GRID2 & GJA3 & PPARG & CD19 & HTR5A & HTR5A & AFAP1L1 \\
\hline SUCNR1 & $\begin{array}{l}H L A^{-} \\
D P B 1\end{array}$ & IRF4 & $B M P 2$ & GNG12 & GRIA2 & $C Y G B$ & $C D 40$ & GJA1 & GRIA2 & CD19 & TMEM88B \\
\hline HTR5A & SUCNR1 & $V W F$ & $I L 1 B$ & GNG7 & $K L F 4$ & $K L F 2$ & GJA1 & CTGF & PPARG & PPARG & HS6ST2 \\
\hline SSTR4 & NPFFR2 & $B M P 2$ & $N P Y$ & GNG11 & PRKCG & STK32B & CTGF & $C D 40$ & $B M P 2$ & $N R P 1$ & CYSLTR2 \\
\hline
\end{tabular}

Table 3. Cox regression results of hub gene

\begin{tabular}{|lllllll|}
\hline gene & \multicolumn{2}{l}{ Univariate cox. } & \multicolumn{4}{c|}{ Multivariable Cox. } \\
\cline { 2 - 5 } & $\beta$ & $\mathrm{HR}(95 \% \mathrm{Cl})$ & $p$-value & $\beta$ & $\mathrm{HR}(95 \% \mathrm{Cl})$ & $p$-value \\
\hline NPY & 0.17 & $1.18(1.10-1.28)$ & $<0.01$ & & & \\
\hline IL1B & 0.21 & $1.23(1.10-1.39)$ & $<0.01$ & & & \\
\hline IL6 & 0.26 & $1.29(1.07-1.56)$ & $<0.01$ & & & \\
\hline BMP2 & 0.18 & $1.20(1.12-1.28)$ & $<0.01$ & & & 0.007 \\
\hline PPARG & -0.21 & $0.81(0.66-0.93)$ & 0.039 & -0.19 & $0.78(0.67-0.91)$ & 0.003 \\
\hline CD19 & 0.19 & $1.21(1.11-1.31)$ & $<0.01$ & 0.14 & $1.15(1.05-1.26)$ & 0.017 \\
\hline GNG13 & 0.29 & $1.34(1.07-1.68)$ & 0.012 & & & \\
\hline CD40 & 0.30 & $1.35(1.17-1.55)$ & $<0.01$ & & & \\
\hline GNG7 & 0.19 & $1.21(1.10-1.32)$ & $<0.01$ & & & \\
\hline GNG12 & 0.35 & $1.42(1.19-1.70)$ & $<0.01$ & 0.23 & $1.25(1.04-1.51)$ & 0.017 \\
\hline
\end{tabular}


Table 4. Cox regression results of independent predictors

\begin{tabular}{|c|c|c|c|c|}
\hline & \multicolumn{2}{|l|}{ Univariate Cox } & \multicolumn{2}{|l|}{ Multivariate Cox } \\
\hline & \multicolumn{2}{|c|}{ Relapse-free survival } & \multicolumn{2}{|c|}{ Relapse-free survival } \\
\hline & $\operatorname{HR}(95 \% \mathrm{Cl})$ & $p$-value & HR (95\% Cl) & $p$-value \\
\hline Age $(0,6]$ & 1 & & & \\
\hline$(6,12]$ & $1.72(0.66-4.48)$ & 0.265 & & \\
\hline$(12,18]$ & $0.91(0.32-2.63)$ & 0.863 & & \\
\hline Sex (female vs male) & $2.10(1.09-4.42)$ & 0.017 & $1.20(0.72-2.02)$ & 0.490 \\
\hline Race (others ${ }^{\mathrm{a}}$ vs White) & $0.34(0.05-2.51)$ & 0.289 & & \\
\hline Eth (Hispanic vs Not) & $1.70(1.02-2,63)$ & 0.204 & & \\
\hline \multicolumn{5}{|l|}{ WBC count $₫ 10^{9} / L \rrbracket$} \\
\hline$\leq 10$ & 1 & & & \\
\hline$(10,100]$ & $1.19(0.34-4.25)$ & 0.780 & & \\
\hline$>100$ & $1.11(0.51-2.42)$ & 0.803 & & \\
\hline Day-29 MRD (>0 vs 0) & $1.70(1.13-2.57)$ & 0.360 & & \\
\hline \multicolumn{5}{|l|}{ CNS classification } \\
\hline CNS1 & 1 & & & \\
\hline CNS2 & $0.86(0,32-2.32)$ & 0.770 & $0.52(0.19-2.29)$ & 0.519 \\
\hline CNS3 & $3.38(1.25-9.10)$ & 0.016 & $5.75(2.01-16.4)$ & 0.001 \\
\hline Trisomy (N v P) & $0.98(0.36-2.67)$ & 0.964 & & \\
\hline MLL (N v P) & $3.57(0.50-25.66)$ & 0.206 & & \\
\hline BMA Blasts Day 8 ( $\geq 6$ vs $₫ 6$ ) & $1.17(0.79-1.71)$ & 0.436 & & \\
\hline BMA Blasts Day 15 ( $\geq 2$ vs $<2)$ & $1.06(0.64-1.76)$ & 0.829 & & \\
\hline BMA Blasts Day 29 (>0) & $0.98(0.64-1.50)$ & 0.930 & & \\
\hline DNA index $\varangle>1$ vs $1 \otimes$ & $1.50(0.61-3.73)$ & 0.386 & & \\
\hline T Cell V B Cell ALL & $2.59(1.18-5.68)$ & 0.017 & $1.63(1.06-2.49)$ & 0.026 \\
\hline Prediction model Risk score & $3.06(1.30-7.19)$ & 0.011 & $1.81(1.16-2.32)$ & 0.046 \\
\hline
\end{tabular}

a, Include black and Native Hawaiian or other Pacific Islander Asian and Unknown;

Table 5. Layered analysis of CD19 gene between T cells and B cells

\begin{tabular}{|llll|}
\hline Non-relapse vs. relapse & Log FC & P-value & FDR \\
\hline B-cells & 2.176 & 0.018 & 0.037 \\
T-cells & -0.318 & 0.184 & 0.367 \\
\hline
\end{tabular}

\section{Figures}




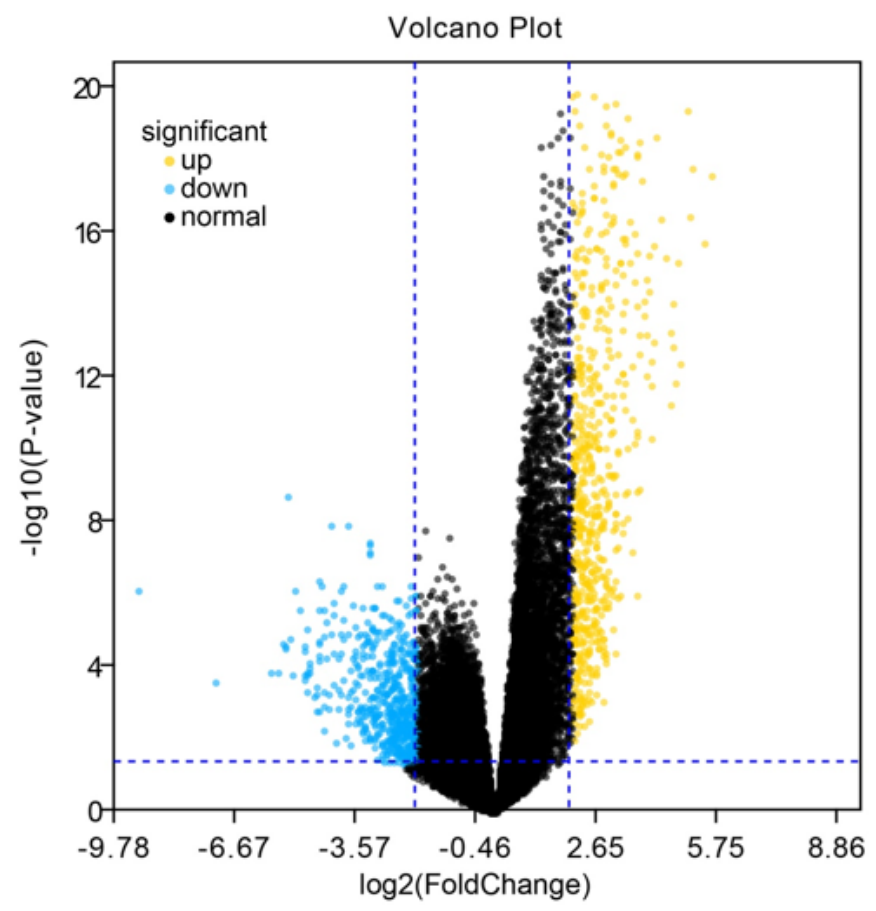

Figure 1

Volcano map of DEGs a yellow represents up-regulated genes, blue represents down-regulated genes, and black represents no statistically significant genetic difference.

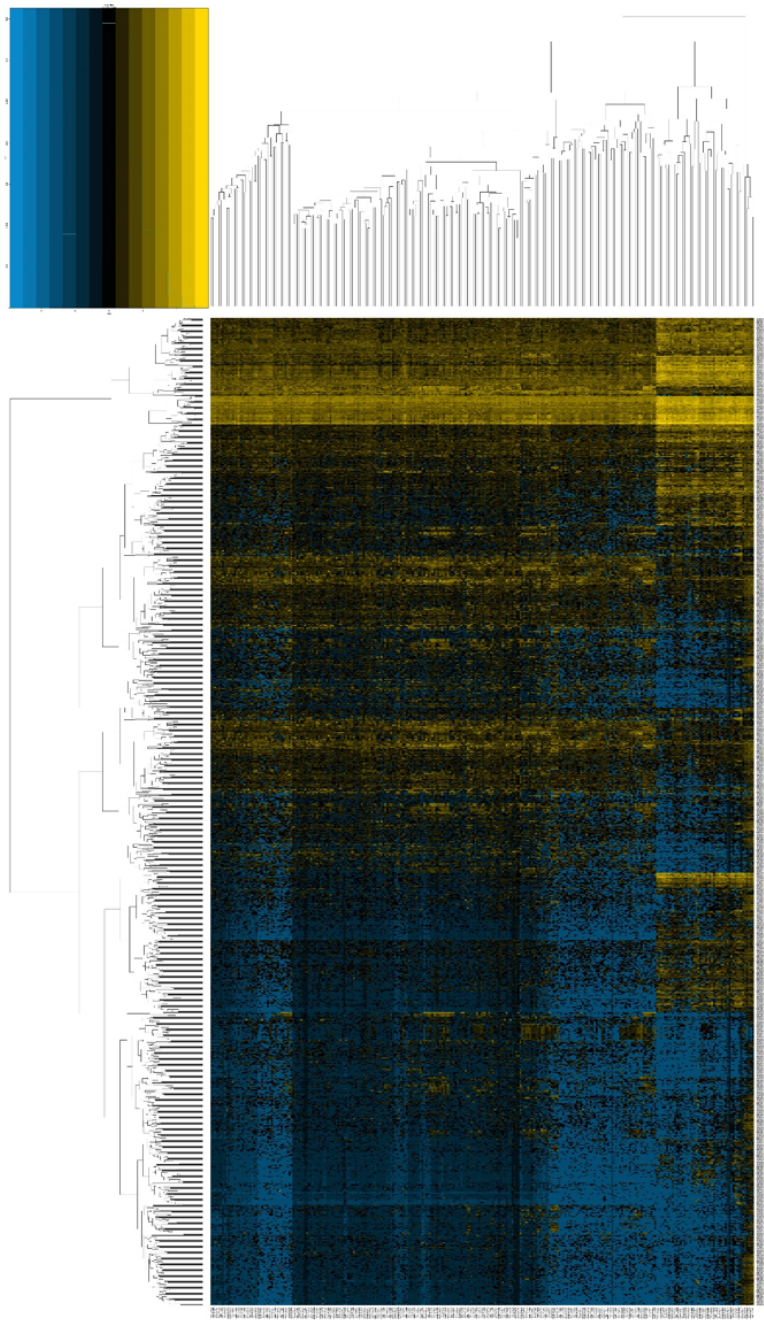


Figure 2

Heat map of DEGs

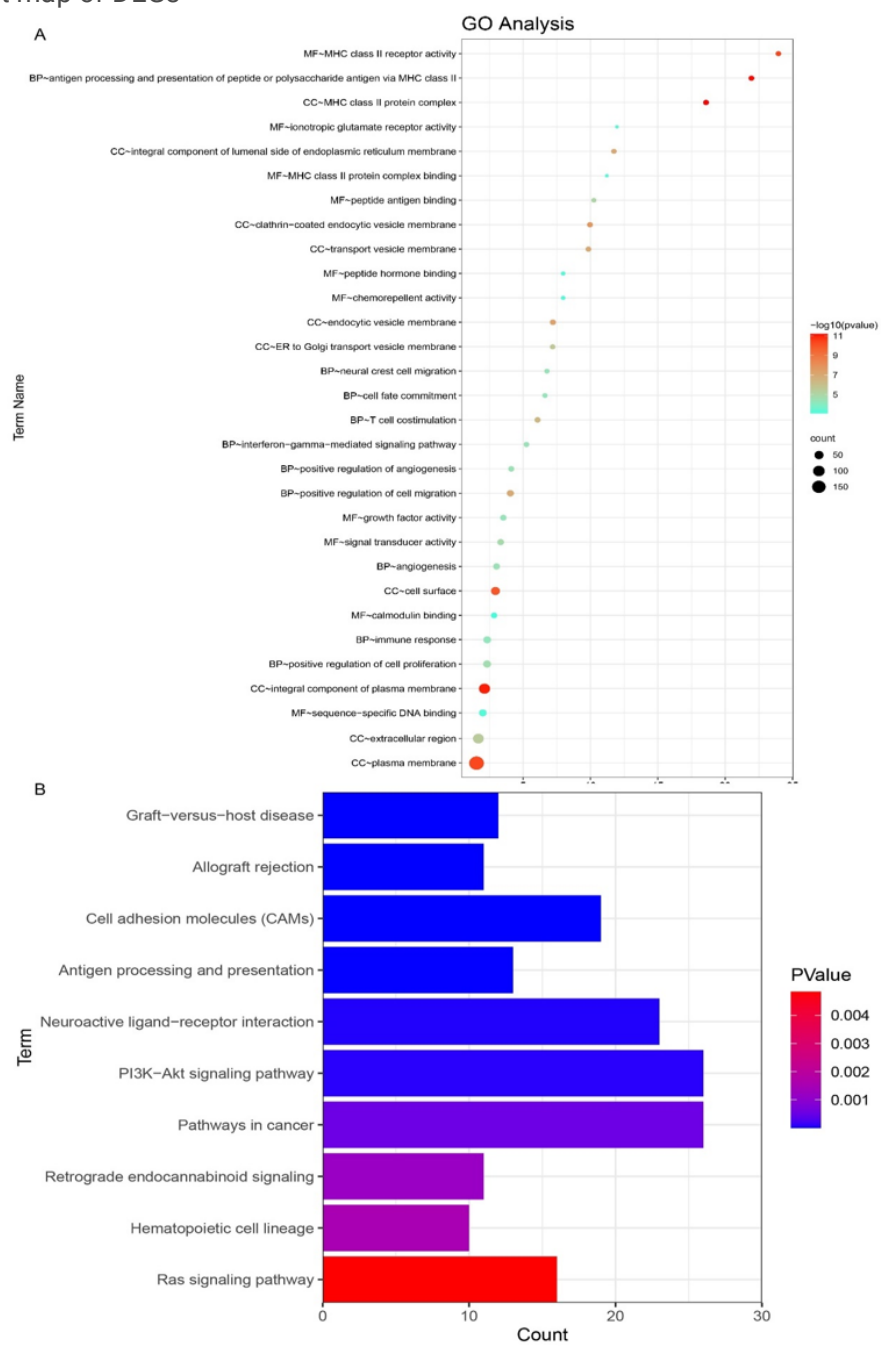

\section{Figure 3}

Gene ontology (A) and KEGG (B) pathway analysis of DEGs The top 10 pathway with the smallest p value were selected for plotting. GO, Gene Ontology; BP, biological process; CC, cell composition; MF, molecular function; KEGG, Kyoto Encyclopedia of Genes and Genomes; 


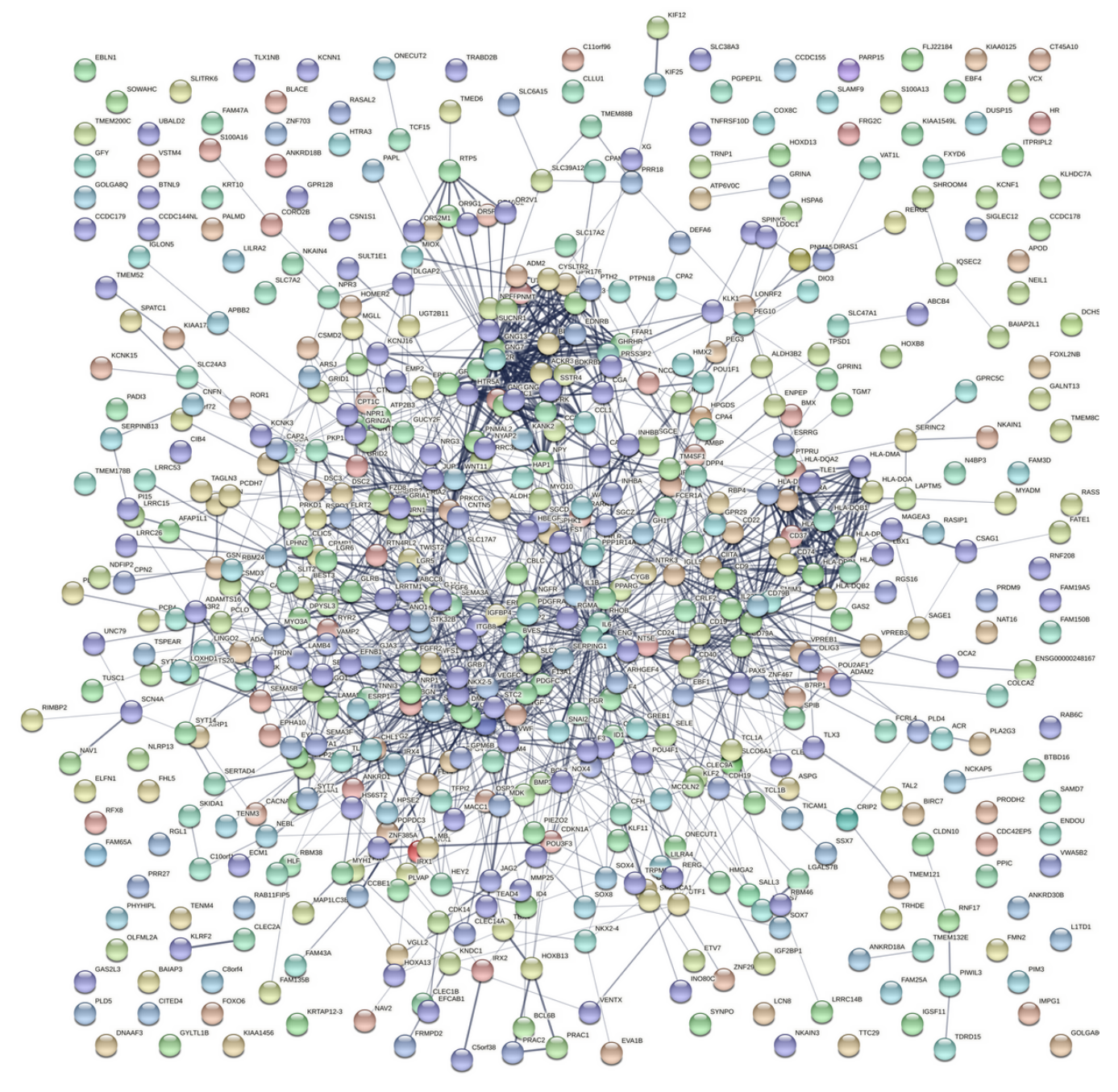

\section{Figure 4}

Differential gene-protein interaction networks. Cut-off value of credibility $>0.7$, received 580 proteins and 647 edges. 


\section{Hub gene determination}

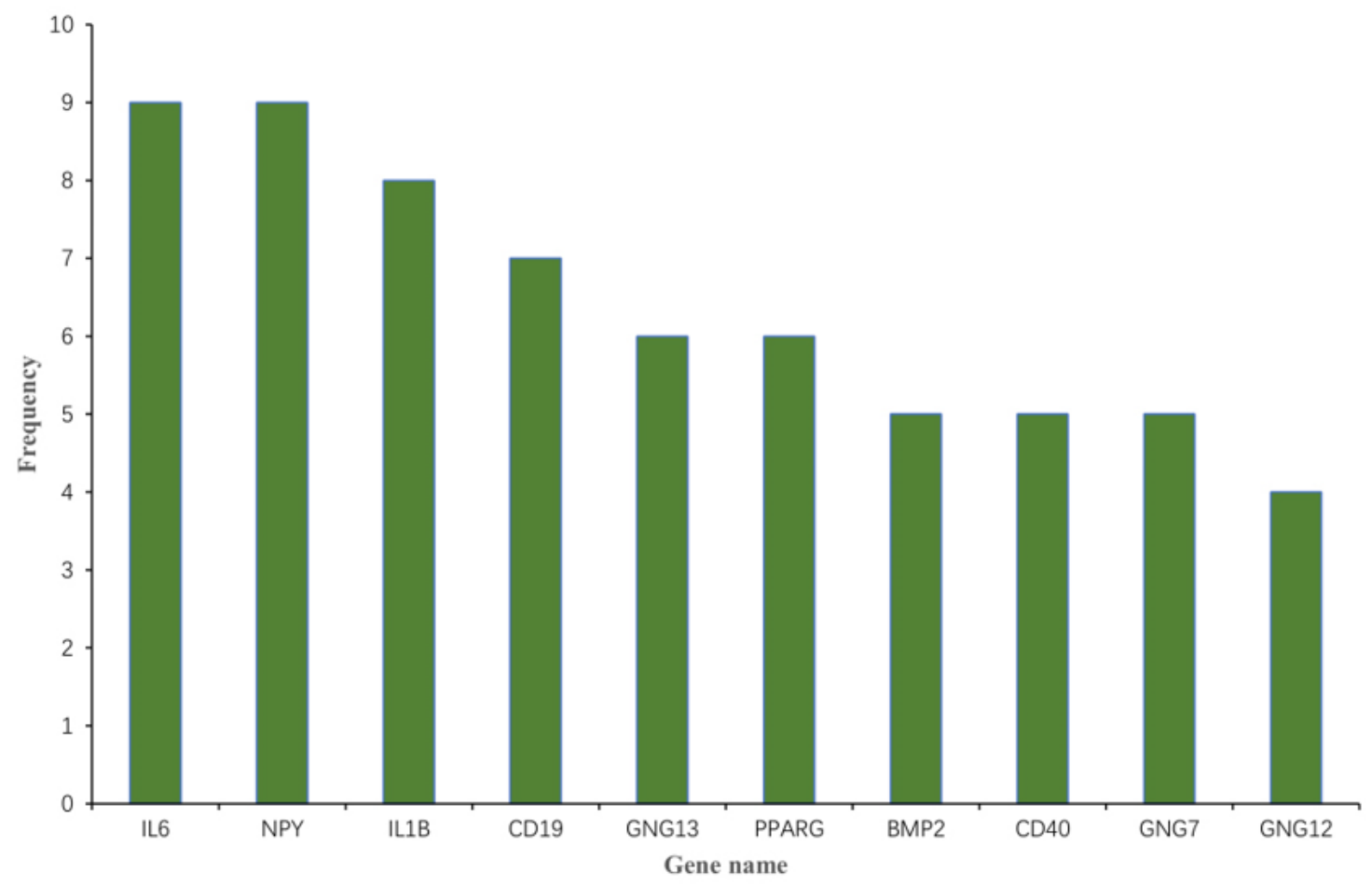

Figure 5

Top 10 genes with frequency. Top 10 genes with the highest occurrence frequency of twelve topological analysis methods were used for frequency statistics.

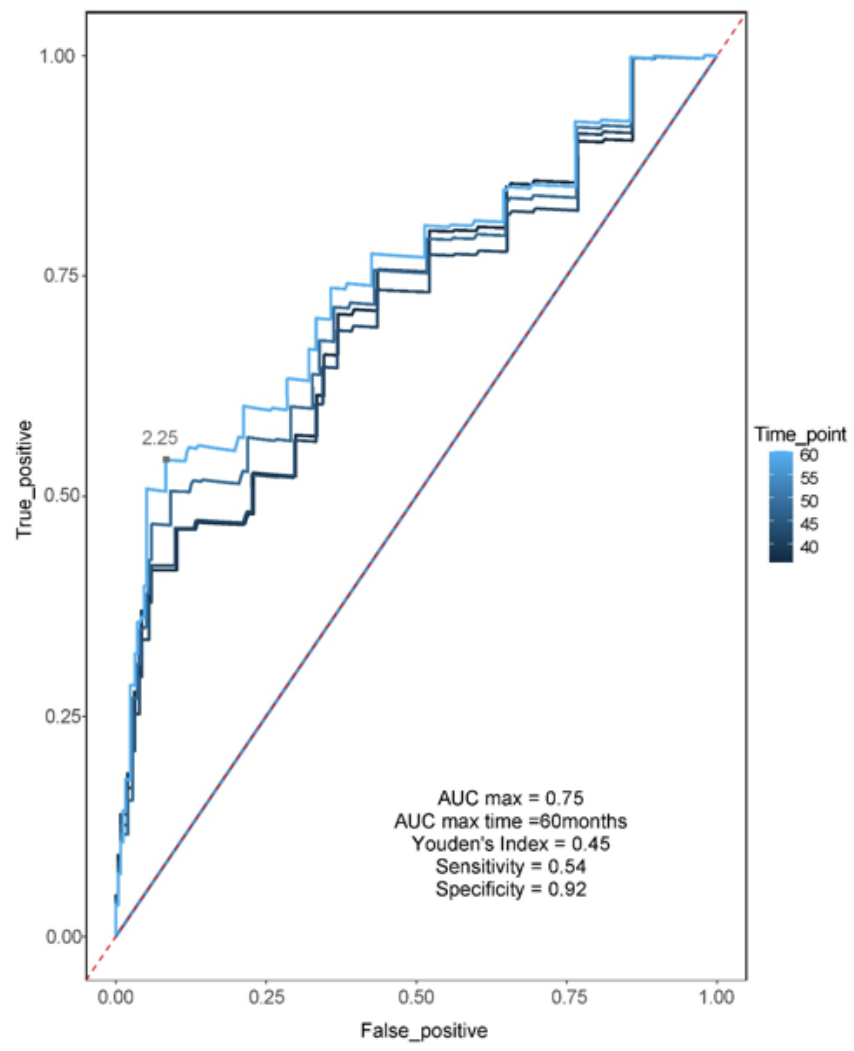

Figure 6

ROC curve of the prediction model. ROC curves at multiple time points across 3-5 years were done in batches to compare and screen out the time point at which the maximum AUC was obtained. 


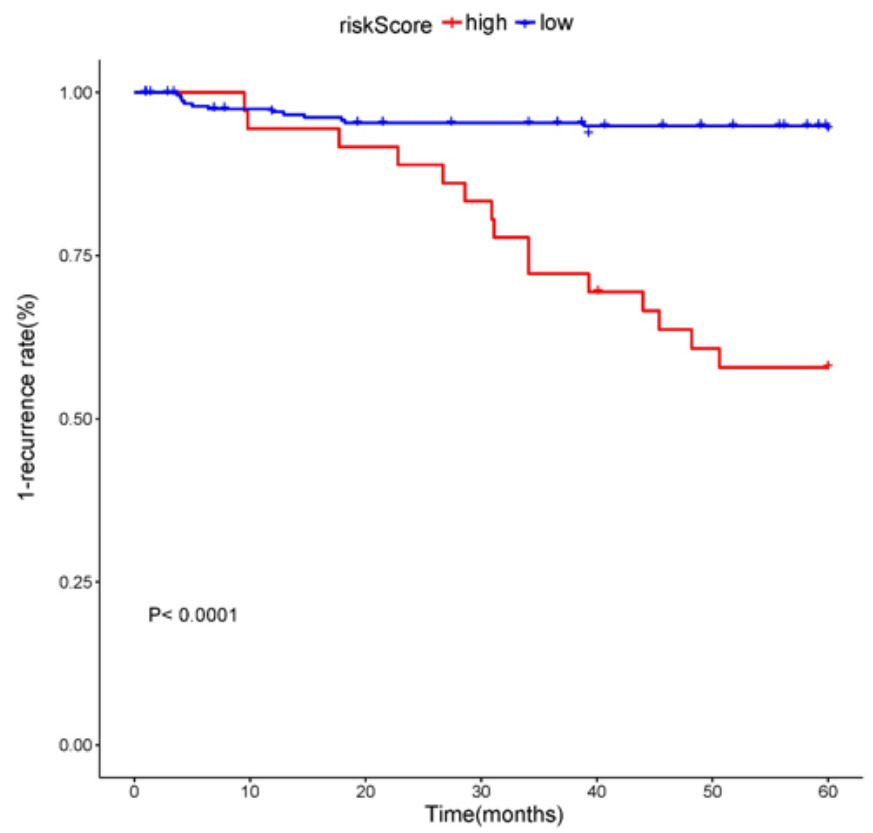

Figure 7

Kaplan-Meier curve of high risk and low-risk group. The true positive and false positive difference maximum points were taken as cut-off of the risk score. Values above this score were defined as a high-risk group, and below this score as a low-risk group

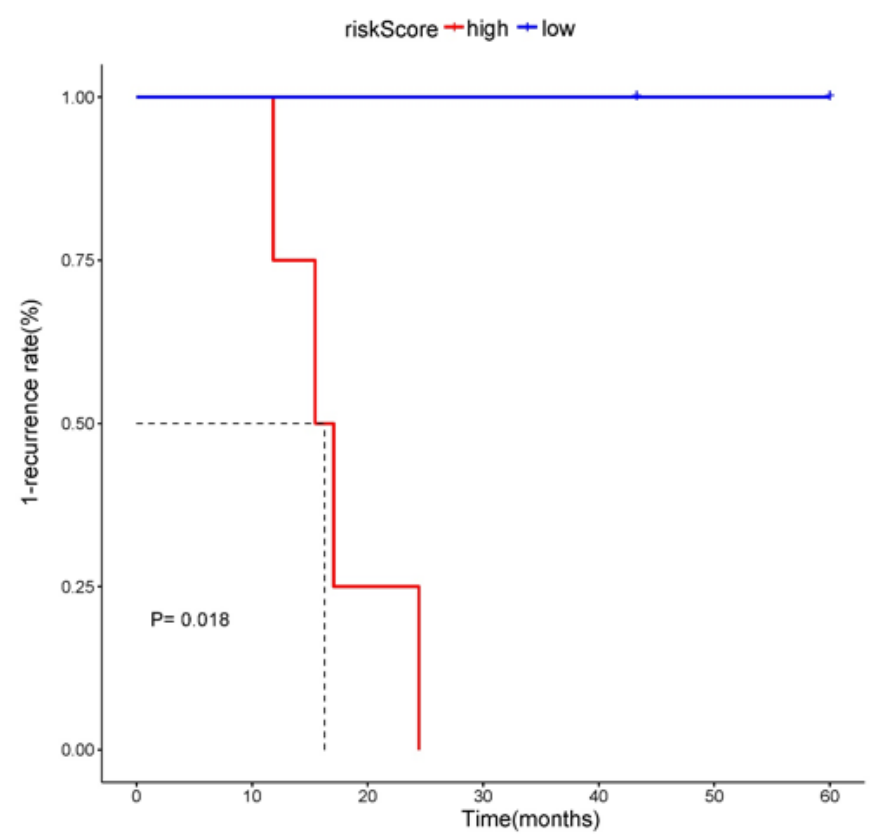

Figure 8

Kaplan-Meier curve of high and low risk of the validation group. The true positive and false positive difference maximum points were taken as cut-off of the risk score. Values above this score were defined as a high-risk group, and below this score as a low-risk group 